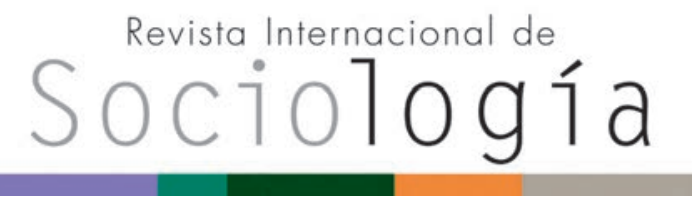

Revista Internacional de Sociología RIS vol. 75 (2), e064, abril-junio, 2017, ISSN-L:0034-9712 doi: http://dx.doi.org/10.3989/ris.2017.75.2.15.44

\section{PRESENTE Y FUTURO DE LA SOCIOLOGÍA DE LA VEJEZ EN ESPAÑA Conclusiones de un estudio Delphi}

Mariano Sánchez Martínez

Universidad de Granada

marianos@ugr.es

ORCID iD: https://orcid.org/0000-0002-1129-9525

JUAN LÓPEZ DOBLAS

Universidad de Granada

jdoblas@ugr.es

ORCID iD: https://orcid.org/0000-0002-0145-355X

Cómo citar este artículo / Citation: Sánchez, M. y J. López Doblas. 2017. "Presente y futuro de la sociología de la vejez en España. Conclusiones de un estudio Delphi”. Revista Internacional de Sociología 75 (2): e064. doi: http://dx.doi. org/10.3989/ris.2017.75.2.15.44

\footnotetext{
Resumen

Estudios internacionales han señalado el insuficiente desarrollo y consolidación de la sociología de la vejez como campo científico y académico distinto. En España, se trata de una especialidad aún bastante invisible y desorganizada, sin establecer plenamente. Con el objetivo de colaborar en la evaluación del presente y el futuro de la sociología de la vejez española se ha llevado a cabo un estudio Delphi con participación de 12 expertos. Los resultados del estudio destacan dos amenazas para la sociología de la vejez española: la percepción de la vejez como etapa del ciclo vital a extinguir y el intrusismo profesional. La invisibilidad de los estudios sociológicos sobre la vejez y la falta de interés por parte de responsables universitarios, agentes políticos y personas mayores constituyen otras amenazas. El principal activo que tiene este campo es la comunidad de sociólogos implicados en el estudio de la vejez.
}

\section{Palabras Clave}

Campo sociológico; Comunidad científica; Envejecimiento; Intrusismo; Invisibilidad.

\section{PRESENT AND FUTURE OF SOCIOLOGY OF OLD AGE IN SPAIN \\ Conclusions from a Delphi Study}

Copyright: @ 2017 CSIC. Este es un artículo de acceso abierto distribuido bajo los términos de la licencia Creative Commons Attribution (CC BY) España 3.0.

Recibido: 16/03/2015. Aceptado: 08/04/2016.

Publicado online: 29/05/2017

\begin{abstract}
International studies have indicated sociology of old age's insufficient development and consolidation as distinct scientific and academic field. In Spain, this speciality is still pretty much invisible, in disarray, and not fully established. Aiming to collaborate in the appraisal of the current and future state of sociology of old age in Spain, a Delphi study with participation of 12 experts has been undertaken. Results highlight two main threats for Spanish sociology of old age, namely perception of old age as a life stage in extinction, and professional encroachment. Invisibility of sociological studies of old age and lack of interest from higher education administrators, policymakers, and older adults themselves are considered threats as well. The main asset in this field is deemed to be the community of sociologists involved in old age studies.
\end{abstract}

\section{KeYWORDS}

Ageing; Encroachment; Invisibility, Scientific Community; Sociological Field. 


\section{INTRODUCCIÓN}

Las preguntas de partida de este artículo son las siguientes: ¿cuál es su situación presente y sus principales retos futuros? y ¿por qué la sociología de la vejez no constituye aún en España un espacio distinto y plenamente establecido dentro de la sociología académica? Para tratar de responder a estas dos interrogantes se puso en marcha, en julio de 2012, el proyecto de investigación Presente y futuro de la sociología de la vejez en España, iniciativa que coincidió con la aparición en Norteamérica de un par de trabajos en los que se abordaban las dos cuestiones citadas. Por un lado, Settersen y Angel (2011a) acababan de editar un nuevo Handbook of Sociology of Aging, y, por otro, Barrett, Redmon y von Rohr (2012) habían sacado a la luz una evaluación del alcance de las investigaciones sobre vejez, envejecimiento y curso vital publicadas en tres revistas académicas de referencia en sociología y psicología. Al igual que nosotros, en último término los autores de ambos textos trataban de contribuir al fortalecimiento de las aproximaciones sociológicas en torno a la vejez y el envejecimiento, vistos la "desconcertante falta de interés en la 'sociología del envejecimiento"' por parte de la sociología dominante (Powell y Hendricks 2009: 86), y el hecho de que los sociólogos parecian ir por detrás de gerontólogos y de especialistas en el curso vital a la hora de estudiar el envejecimiento y sus implicaciones sociales (op. cit. 2012).

¿Son la vejez y el envejecimiento asuntos susceptibles de abordaje sociológico? Sin duda. Ahora bien, tanto en Norteamérica como en Europa Occidental, y para beneficio de la gerontología social, parece que los estudios sociológicos en torno a la vejez y el envejecimiento no han alcanzado un estatus independiente y distinto como campo plenamente establecido dentro de la sociología académica dominante. En la actualidad, la gerontología social, los estudios de curso vital y la sociología de la vejez y del envejecimiento coexisten como formas distintas de investigar el envejecimiento humano en el seno de las ciencias sociales. Sin embargo, esa coexistencia no se produce en igualdad de condiciones: es la gerontología social la que, en efecto, parece llevar la delantera.

En general, se presenta a la gerontología social como campo multidisciplinar "enraizado en la sociología de la edad pero informado por psicólogos, investigadores de políticas y salud públicas, profesionales de la medicina y el trabajo social, demógrafos y economistas, entre otros" (Putney, Alley y Bengtson 2005: 90). La gerontología ha sido considerada como un campo "integrador", que "toma prestado [...] de la sociología" (Bergman 2011: 155). Y se ha llegado a concluir que "la gerontología ha alcanzado la etapa de transformación en una disciplina académica propia" (Lowenstein 2004: 130) en la medida en que ha conseguido avanzar bastante -algo que no parece haber sucedido en el caso de la sociología de la vejez- en el logro de cuatro criterios básicos para ello (Bramwell 1985):

1. Contar con un tema central y transversal a todos sus estudios.

2. Disponer de métodos de investigación propios.

3. Dotarse de una comunidad de expertos capaz de instruirse a sí misma.

\section{Desarrollar una tradición de actividad intelectual.}

En línea con el interés de la gerontología por abarcar todos los esfuerzos disciplinares de investigar el envejecimiento -con la excepción de los que están bajo control de la medicina, de los que se ocupa la geriatría-, la gerontología social intenta cubrir tres tipos de temáticas: (i) las personas envejecidas, (ii) el envejecimiento como proceso de desarrollo, y (iii) el estudio de la vejez "como una dimensión de estructura y comportamiento" (Putney, Alley y Bengtson 2005: 90-91). Esta concepción de la gerontología social considera la sociología de la vejez y el envejecimiento tan solo un subcampo gerontológico.

Putney, Alley y Bengtson (2005), inspirándose en el trabajo de Burawoy (2005), han prestado atención a la cuestión de qué es lo que diferencia a la gerontología social de la sociología. Estos autores concluyen que, de modo distinto a lo que le sucede a la sociología, la gerontología sí es inherentemente multidisciplinar, ya que (i) consiste en un campo mezclado de sociologías profesional, crítica, de las políticas y pública, (ii) entra menos en cruzadas morales, (iii) se dedica tanto a investigación básica como aplicada y, por último, (iv) ha alcanzado la categoría de sociología pública, es decir, una sociología capaz de llegar a un público propio que incluso ella misma ha contribuido a crearse dentro de la academia. Este tipo de análisis relega la sociología de la vejez y el envejecimiento a un segundo plano al considerarla sin más una especie de componente subsumido en la gerontología social. ¿Con qué consecuencias para el desarrollo de los análisis sociológicos en torno a la temática de la vejez y el envejecimiento? Se ha afirmado que tanto la gerontología social como los estudios del curso vital limitan las posibilidades de que la sociología de la vejez llegue a convertirse en un campo científico propio y distinto: la gerontología social, por acaparación (Katz 1996), los estudios de curso vital, por dilución del objeto de estudio más propio de una sociología de la vejez -los órdenes, acciones y sistemas de relaciones sociales asociados a una fase concreta del ciclo vital humano- (Angel y Settersten 2011).

Por lo que respecta a la relación entre la sociología del envejecimiento y los estudios del curso vital, Angel y Settersten (ibíd.: 671) han planteado uno de los argumentos más convincentes acerca de lo que la sociología del envejecimiento ha ganado y ha perdido tras el creciente énfasis puesto en el curso vital: 
"Al tratar de entender 'el envejecimiento y el curso vital', ¿se convierte nuestro campo en algo demasiado grande y amplio de manejar, un campo en el que ocuparse de todas las edades posibles y todas las transiciones posibles en todos los dominios posibles de la vida?". El deseo de conectar múltiples edades algo intrínseco en los estudios del curso vital- obliga a un replanteamiento de la razón de ser y del objeto de lo que en su inicio pretendía ser una sociología centrada exclusivamente en la vejez. Incluso, más allá de la reconsideración de su objeto, Marshall y Bengtson (2011) llaman a un profundo replanteamiento de perspectiva cuando denuncian que los actuales sociólogos del envejecimiento están anclados dentro de sus fronteras disciplinarias y son reacios a teorizar fuera de su zona de confort. Por ello, estos dos autores invitan a los investigadores que trabajan en torno al envejecimiento y al curso vital -sociólogos incluidos- a cruzar las fronteras disciplinares y concluyen que hoy en día sería deseable contar con más estudios inter y transdisciplinares sobre la edad y el envejecimiento.

¿Es la sociología de la vejez un campo de estudio distinto y establecido en la geografía académica y disciplinar? Apoyándonos en las reflexiones que acabamos de repasar, la respuesta es que más bien no; de hecho, atravesamos un momento de reconsideración al respecto. Según lo visto, se puede decir que aprovechando la lógica según la cual el envejecimiento es un proceso con múltiples caras y que, por tanto, precisa un acercamiento multidisciplinar, la gerontología social parece estar abriéndose paso como el auténtico campo académico responsable de los estudios sociales sobre el envejecimiento. Por lo menos, esto es lo que da la impresión de haber sucedido según las interpretaciones norteamericanas. En este orden de cosas, la sociología de la vejez permanece aún bastante invisible e infradesarrollada como campo académico y profesional distinto. Más allá de una mera acumulación de conocimientos procedentes de disciplinas tradicionales como la sociología, la gerontología social ha logrado de alguna manera diluir la capacidad de la sociología de la vejez y del envejecimiento para mantener su identidad como opción fuerte, diferenciada e independiente, para analizar a las personas envejecidas, la vejez, el envejecimiento y las edades. Más aún, los sociólogos no parecen haber demostrado mucha resistencia ante lo que podría considerarse como un proceso ambivalente mediante el cual la gerontología ha contribuido, por un lado, al reconocimiento, pero por otro, al debilitamiento del espacio de especialización específico de la sociología.

En medio de este panorama ambivalente, ¿qué está sucediendo en España con la sociología de la vejez? ¿Hemos logrado constituir un espacio sociológico distinto y establecido en torno al estudio de la vejez y el envejecimiento? En nuestro país, Pinazo y
Sánchez (2005) dataron a mediados de la década de 1970 los primeros trabajos de campo sobre temas de envejecimiento desde una perspectiva sociológica. Por ejemplo, en 1979, Rodríguez publicó un artículo pionero titulado "Perspectiva sociológica de la vejez", con un fuerte énfasis en la gerontología social, en línea con los análisis norteamericanos del momento (Rodríguez 1979). Y un par de años después, Nieto (1981) redactó un breve comentario acerca del trabajo de Rodríguez en el que destacó que, a excepción de los enfoques médicos, los estudios sociales españoles sobre la vejez eran, a comienzo de la década de 1980, una especie de "páramo desierto".

Un hito en el panorama de la investigación española en sociología de la vejez fue el número monográfico publicado por la REIS bajo la dirección de

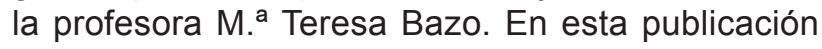
señera, Bazo (1996) explicó no solo que en ese momento los estudios sociológicos sobre la vejez estaban de moda, sino que la sociología de la vejez como subdisciplina dentro de la sociología general había progresado a consecuencia de la preocupación creciente en torno al envejecimiento demográfico, la vejez, la situación de las personas mayores y las potenciales consecuencias del envejecimiento para la economía y las políticas sociales y públicas. De hecho, Bazo (1996) informaba de que, en 1995, el V Congreso Español de Sociología había acogido, por primera vez, un grupo de trabajo sobre sociología de la vejez. Asimismo, por entonces se propuso la constitución de un grupo de trabajo permanente sobre vejez en el seno de la Federación Española de Sociología.

Sin embargo, apenas catorce años después esa situación de apogeo parecía haber cambiado. El X Congreso Español de Sociología no contó con una sección específica dedicada a la vejez; todas las comunicaciones relacionadas con la vejez y el envejecimiento fueron integradas dentro del grupo de trabajo 'Sociología de la Edad y del Ciclo Vital'. En ese Congreso se realizaron un total de 27 presentaciones relacionadas con la vejez y el envejecimiento -en 1996, la cifra se había acercado a 60 (Bazo 1996) - y en 2013 esa cifra se redujo a catorce, según la Federación Española de Sociología (FES, s.f.). Más recientemente hemos sido testigos de un hecho que se podría interpretar como un paso más en la desinstitucionalización del campo sociológico de la vejez: en noviembre de 2015, el Comité Ejecutivo de la FES tomó la decisión de suprimir el grupo 'Sociología de la Edad y del Ciclo Vital' de cara al Congreso Español de Sociología previsto en 2016.

Ante la falta de repasos sistemáticos sobre el estado de este campo en España, y a efectos de contextualizar la pertinencia del estudio Delphi que sigue, se llevó a cabo una revisión de los artículos que han aparecido en cinco de las revistas españolas de sociología de mayor impacto académico -RIS 
(Revista Internacional de Sociología), REIS (Revista Española de Investigaciones Sociológicas), Papers, Política y Sociedad, y RES (Revista Española de Sociología)- desde su fundación hasta el último número disponible en 2014 (salvo en el caso de la RIS, cuya revisión se ha restringido a lo publicado desde el inicio de su tercera época, en 1992). Se seleccionaron los textos sobre temáticas relacionadas con la vejez, el envejecimiento o las personas mayores; el criterio de selección seguido fue que en su título aparecieran expresamente términos como vejez, ancianos, tercera edad, mayores, personas mayores, envejecimiento o algún otro -como jubilación, abuelos o residencias- que de modo implícito aludiera a nuestro objeto de estudio. El análisis de los 61 artículos identificados nos permitió llegar a las tres conclusiones siguientes:

a) Continuidad de trabajos publicados. Hasta 1989 aparecieron tan solo siete, todos en la misma revista; en la década de 1990, sin embargo, un total de 29 , muchos de ellos formando parte del número monográfico "Envejecimiento y Sociedad", que editó Papers en 1992 -volumen 40-, o del monográfico "Sociología de la Vejez", publicado por la REIS en 1996 -número 73-. De 2000 a 2014 se han publicado 25 nuevos artículos -solo en 2001 y 2005 no se incluyó ningún texto; en el resto de los años, siempre se ha publicado algo-.

b) Cambio en las temáticas abordadas. Durante las dos últimas décadas del siglo XX parecieron interesar sobremanera los aspectos negativos relacionados con el envejecimiento y las personas mayores -la soledad, el conflicto entre generaciones, la institucionalización, la dependencia, el suicidio, etc.-, al tiempo que se planteó un incipiente debate sobre la naturaleza de la sociología de la vejez como área específica de la disciplina; pero ese debate no tuvo continuidad y desapareció. Desde el año 2000 ha disminuido la predilección por tratar los problemas que popularmente suelen asociarse con las personas mayores y han ido emergiendo en la agenda aspectos relacionados con la vida cotidiana, el género o las relaciones intergeneracionales. Asimismo, el término "anciano" ha dejado de emplearse, si bien anteriormente tuvo un uso relativamente habitual.

c) Escasez de sociólogos dedicados específicamente a este campo. La autoría de una parte considerable de los artículos corresponde a investigadores que suelen dedicarse -además- a estudiar otras áreas de la sociología como la población, la familia o la educación, por ejemplo; es decir, que entre los autores son escasos los expertos reconocidos con una trayectoria investigadora firme, continuada y monográfica en el campo de la sociología de la vejez y del envejecimiento.

El marco institucional y académico descrito -tanto el internacional como el nacional-, que podríamos calificar de incierto y cambiante, invita a analizar la situación de la sociología de la vejez española; particularmente, su posición frente a otros campos de las ciencias sociales que, desde diversos ángulos, trabajan sobre el envejecimiento y/o las personas mayores. Sin embargo, hasta ahora nadie se ha ocupado de responder a estas cuestiones mediante un trabajo de investigación, algo que sí afronta el presente artículo, basado en los resultados de un estudio Delphi que ha tratado de cubrir ese vacío al ofrecer a la comunidad de expertos implicados en ese campo académico y disciplinar una posibilidad para la reflexividad mediante al aprovechamiento de las ventajosas condiciones de ese tipo de estudios, que permiten "abordar la complejidad y la incertidumbre en un área en la que el conocimiento es imperfecto, donde no existen respuestas correctas o hechos incontestables" (Donohoe y Needham 2009: 41).

En este sentido, se puso en marcha el proyecto de investigación "Presente y futuro de la sociología de la vejez en España". Y para abordar un asunto sobre el que la literatura es prácticamente inexistente, se pensó que lo más eficaz sería empezar consultando directamente a quienes se perciben como situados en el campo. En consecuencia, el proyecto adoptó como objetivo fundamental conocer los puntos de vista de investigadores y docentes implicados en la sociología de la vejez española al respecto de la situación de su propio campo profesional. ¿Por qué centrar la investigación en ese grupo? Por dos razones: en primer lugar, por su posición interna al campo, privilegiada a la hora de valorar la situación y el desarrollo del mismo; en segundo lugar, por la posibilidad de reconocimiento mutuo entre dichos profesionales que la propia investigación podría facilitar, abriendo así la puerta a posibles acciones conjuntas a raíz de los resultados finales obtenidos. Facilitar el intercambio de visiones y posibilitar el consenso entre estos investigadores y docentes en torno a la situación actual y futura del campo -se pensó- aportaría información valiosa para comprender la situación por la que atraviesa este ámbito académico en nuestro país y para descubrir los retos fundamentales a los que se ha de enfrentar en los próximos años. Formulado este objetivo, utilizar la técnica Delphi pareció la opción más adecuada, pues lo que interesaba era motivar la reflexión y la reflexividad de los actores más directamente implicados en el campo a estudiar. Con tal fin se planteó el esquema metodológico que pasamos a detallar.

\section{Método}

Hsu y Sandford (2007) han explicado que la técnica Delphi aprovecha la comunicación grupal entre los integrantes de un panel de expertos para abordar un asunto específico, no con la mera finalidad de describirlo, sino para preguntarse sobre cómo ese asunto podría y debería desarrollarse en el futuro. Por tanto, se trata de una técnica de pronóstico más 
que de diagnóstico. En este caso, esta especificidad de la técnica encaja con nuestra intención de preguntarnos, no solo por el presente, sino por las perspectivas de desarrollo que la sociología de la vejez tiene en nuestro país y por lo que, en consecuencia, sería necesario hacer al respecto. Además, los estudios Delphi ya han sido utilizados en numerosas ocasiones en trabajos de corte sociológico (Acevedo-Polakovich et al. 2011; Wittman, Beckie y Hergesheimer 2012). Y también contamos con precedentes del uso específico de esta técnica para el estudio de cuestiones relacionadas con el envejecimiento humano (Ferri et al. 2006; Huenchuan 2009; Hohn 2010; Jeste et al. 2010; Alfageme, García y Viñado 2014).

Jeste et al. (2010) reconocen que esta técnica, considerada como un proceso para posibilitar el consenso en torno a un tema, tiene ventajas: facilita el anonimato y la participación de personas geográficamente dispersas, y descarta el efecto halo, típico en el proceso de un panel cara a cara. Ambas ventajas se ajustaban bien a la situación de los posibles expertos participantes en nuestro caso particular.

Según Bush y Jones (2010), son los investigadores quienes han de llevar a cabo la selección de los participantes, de forma discrecional, teniendo en cuenta el contexto de la investigación y la capacidad que, desde su punto de vista, puedan tener los participantes para aportar su visión. Los criterios de inclusión establecidos para participar en el presente estudio fueron dos: (i) tener experiencia investigadora en sociología de la vejez y (ii) cumplir con una segunda condición de entre las dos siguientes:

- Haber obtenido la licenciatura/grado o el doctorado en sociología.

- Contar con experiencia docente universitaria en el área de conocimiento de sociología.

La Tabla 1 representa, de forma esquemática, las cifras fundamentales relativas al calendario del proceso y a los expertos participantes en el mismo.
Existen precedentes de aplicación de la técnica Delphi a partir de paneles compuestos por diez expertos (Hsu y Sandford 2007; Walker 2011), lo que coloca nuestro caso, con 12 panelistas, dentro del margen aceptable. De hecho, se ha subrayado que los paneles de pequeño tamaño suelen permanecer intactos con más probabilidad a lo largo de todo el proceso y pueden lograr un resultado fiable, en especial cuando, como es el caso, los expertos han sido seleccionados mediante estrictos criterios de inclusión y cuentan con niveles similares de cualificación (Atkins, Tolson y Cole 2005). La Tabla 2 muestra las características fundamentales del perfil de los 12 expertos (5 hombres y 7 mujeres) que finalmente cumplimentaron los tres cuestionarios de los que constó el proceso.

La investigación se organizó en tres cuestionarios cuyo envío, recogida y análisis se extendió entre julio de 2012 y junio de 2013, por encima del mínimo de 45 días para el periodo creación-análisis en cada ronda del proceso según la literatura al respecto (Hohn 2010). Tras un primer cuestionario de carácter exploratorio se analizaron todas las respuestas recogidas y, con esa información, se preparó el segundo cuestionario. En él, los panelistas pudieron acceder a sus propias consideraciones y a las del resto de participantes, así como conocer el grado de consenso emergente en torno a los siete temas en los que en un inicio se organizó la investigación (ver Tabla 3).

Por último, en un tercer cuestionario se les pidió a nuestros expertos que ordenasen sus puntos de vista a modo de ranking final. En este cuestionario se les facilitó, en dos formatos distintos, información relativa a los resultados del cuestionario anterior. Por un lado, en los ranking procedentes de otro previo con selección, los enunciados a ordenar aparecían listados en orden decreciente de importancia de acuerdo con las opiniones expresadas por los panelistas en la segunda ronda del estudio. Por otro lado, en el caso de los ranking sin selección previamente

Tabla 1.

Calendario y expertos participantes

\begin{tabular}{|c|c|c|c|c|c|c|}
\hline Cuestionario & $\begin{array}{l}\text { Recogida de } \\
\text { datos }\end{array}$ & Destinatarios $(n)$ & Respuestas (n) & $\begin{array}{c}\text { Experiencia } \\
\text { investigadora }(n)\end{array}$ & $\begin{array}{l}\text { Licenciatura/ } \\
\text { Grado } \\
\text { Sociología (n) }\end{array}$ & $\begin{array}{l}\text { Experiencia } \\
\text { docente }(n)\end{array}$ \\
\hline $1^{0}$ & $\begin{array}{c}\text { julio-octubre } \\
2012\end{array}$ & 22 & 13 & \multirow{2}{*}{13} & \multirow{2}{*}{13} & \multirow{2}{*}{12} \\
\hline $2^{\circ}$ & $\begin{array}{c}\text { marzo-mayo } \\
2013\end{array}$ & 13 & 12 & & & \\
\hline $3^{\circ}$ & $\begin{array}{l}\text { mayo-junio } \\
2013\end{array}$ & 12 & 12 & 12 & 12 & 11 \\
\hline
\end{tabular}

Fuente: Elaboración propia. 
Tabla 2.

Características de los expertos

\begin{tabular}{lcc}
\hline \multicolumn{1}{c}{ Característica } & Sí $(\mathrm{n})$ & No $(\mathrm{n})$ \\
\hline $\begin{array}{l}\text { Licenciatura / Grado } \\
\text { Sociología }\end{array}$ & 10 & 2 \\
\hline $\begin{array}{l}\text { Doctorado } \\
\text { Sociología }\end{array}$ & 10 & 2 \\
\hline $\begin{array}{l}\text { Docencia } \\
\text { universitaria }\end{array}$ & 11 & 1 \\
\hline $\begin{array}{l}\text { Experiencia en } \\
\text { investigación }\end{array}$ & 12 & - \\
\hline $\begin{array}{l}\text { Experiencia en } \\
\text { intervención }\end{array}$ & 6 & 6 \\
\hline
\end{tabular}

Fuente: Elaboración propia.

Tabla 3.

Temas de la investigación

\begin{tabular}{ll}
\hline \multicolumn{1}{c}{ Tema } & \multicolumn{1}{c}{ Concepto } \\
\hline Debilidades & $\begin{array}{l}\text { Elementos internos al campo de la sociología } \\
\text { de la vejez en España que constituyen puntos } \\
\text { débiles, es decir, fragilidades, flaquezas. }\end{array}$ \\
\hline \multirow{3}{*}{ Fortalezas } & $\begin{array}{l}\text { Elementos internos del campo de la sociología } \\
\text { de la vejez en España que suponen justamente } \\
\text { lo contrario de las debilidades: los puntos } \\
\text { fuertes, los activos, aquello que le da robustez } \\
\text { al campo. }\end{array}$ \\
\hline Razones de & $\begin{array}{l}\text { Razones, motivos, causas, explicaciones de } \\
\text { por qué la sociología de la vejez en España } \\
\text { como especialidad académica se encuentra } \\
\text { actual } \\
\text { en su situación actual. Deben aludir, de un } \\
\text { modo u otro, a factores que teniendo su } \\
\text { origen en el pasado nos ayudan a explicar } \\
\text { el presente. }\end{array}$ \\
\hline Amenazas & $\begin{array}{l}\text { Elementos externos al campo de la sociología } \\
\text { de la vejez en España que lo pueden poner } \\
\text { en situación de peligro y atentar contra su } \\
\text { existencia y desarrollo futuros. }\end{array}$ \\
\hline \multirow{2}{*}{ Oportunidades } & $\begin{array}{l}\text { Elementos externos al campo de la sociología } \\
\text { de la vejez en España que pueden ayudar a } \\
\text { impulsarlo y fortalecerlo de cara al futuro. }\end{array}$ \\
\hline \multirow{2}{*}{ Estrategias } & $\begin{array}{l}\text { Accionesfuturas allevara cabo en la sociología } \\
\text { de la vejez en España como especialidad } \\
\text { académica para lograr su desarrollo. }\end{array}$ \\
\hline & $\begin{array}{l}\text { Contenidos de la agenda docente e } \\
\text { investigadora de la sociología de la vejez en } \\
\text { España como especialidad académica. }\end{array}$ \\
\hline
\end{tabular}

Fuente: Elaboración propia.

utilizados en el segundo cuestionario, además de la colocación de los enunciados según su importancia decreciente, se indicó el grado de consenso alcanzado hasta el momento según la interpretación acordada de los valores del estadístico $W$ de Kendall (ver Tabla 4, más abajo). Siguiendo a Okoli y Pawloski (2004), en este último cuestionario solo se incluyeron los ranking con W menor de 0,7 -se entiende que un valor mayor de $\mathrm{W}$ indica que ya se ha alcanzado un grado de consenso suficiente-. Cada panelista recibió, junto con el tercer cuestionario, copia de sus res- puestas al segundo cuestionario para facilitarle así el recuerdo de sus últimas opiniones aportadas al proceso (Sahin 2010). Tanto en el cuestionario segundo como en el tercero se incluyó una pregunta abierta en cada sección temática invitando a los expertos a realizar cualquier comentario al respecto.

Por lo que se refiere al análisis, en el caso del primer cuestionario fue eminentemente cualitativo, con combinación de interpretación deductiva e inductiva. Tras elaborar un libro de códigos provisional (Hruschka et al. 2004; Saldaña 2009), compuesto por 22 códigos -12 relacionados con el marco conceptual y 10 con la literatura sobre el tema- los investigadores procedieron, por separado, a codificar. El proceso de codificación se realizó tomando como patrón el análisis temático según lo explicaron Braun y Clark (2006) y lo aplicó Richardson (2011). La participación de dos codificadores en este proceso, puestos de acuerdo en aplicar de modo independiente pero consistente unas mismas reglas de codificación, asegura una mayor fiabilidad del análisis (Brink 1987; Harris, Pryor y Adams 1997; Hruschka et al. 2004).

La fiabilidad de esta primera codificación y de las siguientes fue medida mediante el estadístico de contraste Kappa de Cohen. Para la interpretación de este estadístico se adoptó un criterio aceptado en la literatura (Landis y Koch 1977; Harris, Pryor y Adams 1997) según el cual un valor de Kappa superior a 0,81 indica que la codificación ha sido altamente fiable. El valor final de Kappa alcanzado fue de 0,89. Todas las codificaciones contradictorias producidas en el proceso fueron discutidas y consensuadas por los investigadores (Graneheim y Lundman 2004).

En el segundo cuestionario se aplicaron procedimientos de análisis distintos para las preguntas tipo Likert y para aquéllas en formato ranking. En el caso de las primeras, y siguiendo la propuesta Hohn (2010), se estableció de antemano que el consenso se alcanzaría cuando un $75 \%$ o más de las respuestas se acumulasen en torno a 'Totalmente en desacuerdo/Bastante en desacuerdo' o 'Bastante de acuerdo/Totalmente de acuerdo'.

En el caso de las preguntas tipo ranking sin exigencia de selección previa de enunciados a ordenar, y adoptando la propuesta de Schmidt (1997), para cada enunciado se calculó la media de las posiciones de ranking asignadas - sin dar importancia a la distancia entre tales medias sino a la posición anterior o posterior de las mismas- y, a continuación, para el conjunto de enunciados agrupados en un misma pregunta, se valoró el grado de consenso mediante el valor del estadístico W de Kendall, interpretado según se indica en la Tabla 4.

Casi todas las preguntas del último cuestionario tenían formato ranking; por tanto, el análisis de las mismas se centró fundamentalmente en calcular, para cada una de ellas, el valor del estadístico W de 
Kendall, comprobar el grado de los consensos finales alcanzados y, en lo posible, compararlos con los obtenidos tras la segunda ronda del estudio.

Tabla 4.

Interpretación del valor de $W$ de Kendall

\begin{tabular}{ll}
\hline $\mathbf{W}$ Kendall & Interpretación \\
\hline $\mathbf{0 , 1} \mathbf{W}<\mathbf{0 , 3}$ & Consenso muy débil \\
\hline $\mathbf{0 , 3} \mathbf{W}<\mathbf{0 , 5}$ & Consenso débil \\
\hline $\mathbf{0 , 5} \leq \mathbf{W}<\mathbf{0 , 7}$ & Consenso moderado \\
\hline $\mathbf{0 , 7} \mathbf{W}<\mathbf{0 , 9}$ & Consenso fuerte \\
\hline $\mathbf{W} \geq \mathbf{0 , 9}$ & Consenso extraordinariamente fuerte \\
\hline
\end{tabular}

Fuente: Schmidt 1997: 767.

\section{Resultados Y DISCUSIÓN}

Como se muestra a continuación, en la Tabla 5, tan solo en cinco aspectos de tres de los temas amenazas, oportunidades y fortalezas- en los que se articuló el estudio (ver listado completo de temas en Tabla 3) nuestros panelistas llegaron a un consenso moderado o superior ( $W$ Kendall $\geq 0,5$ ). Según los parámetros establecidos para medir el consenso, éste fue débil $(0,3 \leq W<0,5)$, muy débil $(0,1 \leq W<0,3)$ o inexistente $(W=0)$ en los cuatro temas restantes.

Comencemos hablando del aspecto en torno al cual se produjo el mayor consenso. Según se detalla en la Tabla 6, el consenso más alto -el valor de W de Kendall pasó de 0,2 en el segundo cuestionario

Tabla 5.

Grados de consenso por temas y aspectos

\begin{tabular}{|c|c|c|}
\hline Tema & Relacionado con... (aspecto) & W Kendall \\
\hline \multirow{4}{*}{ Debilidades } & Temática & 0,3 \\
\hline & Invisibilidad del campo & 0,3 \\
\hline & Establecimiento de las fronteras de la sociología de la vejez & 0,2 \\
\hline & Interés en torno al campo & 0,1 \\
\hline \multirow[t]{2}{*}{ Fortalezas } & $\begin{array}{l}\text { Comunidad de agentes integrantes de este campo: investigadores, docentes y } \\
\text { sociólogos/as profesionales }\end{array}$ & 0,5 \\
\hline & Recursos humanos o de otro tipo de los que dispone la sociología de la vejez & 0,3 \\
\hline \multirow{6}{*}{$\begin{array}{l}\text { Razones de la situación } \\
\text { actual }\end{array}$} & Debilidades arrastradas por el campo desde el pasado & 0 \\
\hline & Temática del campo & 0,1 \\
\hline & Interés por la sociología de la vejez & 0,4 \\
\hline & Establecimiento de fronteras & 0,2 \\
\hline & $\begin{array}{l}\text { Comunidad de agentes integrantes de este campo: investigadores, docentes y } \\
\text { sociólogos/as profesionales más o menos capaces de aprender por sí mismos, tomar } \\
\text { sus propias decisiones y dotarse de sus propias reglas }\end{array}$ & 0,2 \\
\hline & $\begin{array}{l}\text { Amenazas que vienen del pasado, es decir, factores externos al campo que lo han } \\
\text { venido poniendo en situación de peligro y, desde fuera del mismo, han atentado contra } \\
\text { su existencia y desarrollo }\end{array}$ & 0,4 \\
\hline \multirow{5}{*}{ Amenazas } & Recursos (humanos y de todo tipo) disponibles & 0,1 \\
\hline & Invisibilidad de la sociología de la vejez & 0,5 \\
\hline & Desinterés en torno a la sociología de la vejez & 0,5 \\
\hline & Establecimiento de las fronteras del campo & 0,3 \\
\hline & Temática de la que se ocupa la sociología de la vejez & 0,7 \\
\hline \multirow{3}{*}{ Oportunidades } & Temática de la que se ocupa & 0,2 \\
\hline & Interés suscitado en torno a la sociología de la vejez & 0,5 \\
\hline & Agenda investigadora y docente & 0,3 \\
\hline \multirow{6}{*}{ Estrategias } & Temática de la que se ocupa & 0,4 \\
\hline & Agenda investigadora & 0,2 \\
\hline & Método & 0,2 \\
\hline & $\begin{array}{l}\text { Comunidad de agentes (investigadores, docentes y sociólogos/as } \\
\text { profesionales) que integran el campo }\end{array}$ & 0,4 \\
\hline & $\begin{array}{l}\text { Sociología profesional, es decir, la capacidad para } \\
\text { articular métodos, conocimiento, marcos conceptuales y preguntas básicas } \\
\text { orientadores de sus programas de investigación del campo }\end{array}$ & 0 \\
\hline & Invisibilidad & 0 \\
\hline Agenda & Asuntos de la agenda docente e investigadora de la sociología de la vejez española & 0,2 \\
\hline
\end{tabular}


a 0,7 en el tercero-, se alcanzó con relación a las amenazas futuras para la sociología de la vejez en virtud de su temática:

Tabla 6.

Amenazas futuras y temática de la sociología de la vejez

Amenazas futuras relacionadas con la temática de la sociología de la vejez (W Kendall: $\mathbf{0 , 7}$ )

\begin{tabular}{|c|c|c|c|}
\hline & $\mathrm{n}$ & Media & $\begin{array}{l}\text { Desviación } \\
\text { típica }\end{array}$ \\
\hline $\begin{array}{l}\text { El sector de la vejez se } \\
\text { considera 'cuerpo a extinguir' } \\
\text { y, en consecuencia, se } \\
\text { prefiere centrar el esfuerzo } \\
\text { investigador en otros grupos. }\end{array}$ & 12 & 1,92 & 289 \\
\hline $\begin{array}{l}\text { De la vejez todo el mundo } \\
\text { se atreve a hablar. }\end{array}$ & 12 & 1,08 & ,289 \\
\hline
\end{tabular}

Fuente: Elaboración propia.

Detrás de estas dos amenazas podrían estar dos de las percepciones que citamos más arriba cuando nos referimos al estado de la sociología de la vejez en Norteamérica y España. Por un lado, que la vejez sea un cuerpo en proceso de extinción hace pensar que, en efecto, la sociología de la vejez puede ser un campo en busca de objeto de estudio -y ya hemos visto que el envejecimiento y el curso vital parecen bien colocados en ese proceso de búsqueda-. Por otro lado, afirmar que todo el mundo se atreve a hablar de la vejez apunta a la amenaza que supone el que otros campos y expertos, con más o menos conocimiento especializado al respecto, se hayan instalado en el espacio inicialmente ocupado por esta particular sociología. No en vano, al abordar el asunto de cuáles son las razones de la situación actual de la sociología de la vejez, uno de nuestros expertos se refirió expresamente a "la permisividad hacia el intrusismo profesional" (Experto 1).

Sigamos con las amenazas porque este tema fue el que, con diferencia, suscitó más consenso en el grupo de panelistas. En concreto, en otros dos posibles aspectos amenazantes -la invisibilidad de la sociología de la vejez y el desinterés externo en torno a ella-, se alcanzó un grado de consenso moderado (ver Tablas 7 y 8). En ambos aspectos el grado de acuerdo en el segundo cuestionario había sido de 0,2 (W Kendall) pero alcanzó 0,5 en la última ronda del proceso. Estos incrementos en los valores de $\mathrm{W}$ confirman que, en nuestro estudio, la técnica Delphi realmente posibilitó en algunos casos un progresivo aumento del consenso entre los expertos implicados.

El concepto de invisibilidad alude al posible carácter no visible, no perceptible de la sociología de la vejez en España como especialidad académica; dicho de otro modo, esta amenaza invitaría a preguntarnos si estamos ante un campo que sí, existe, pero al que no se le percibe ni se le reconoce como un campo distinto, con espacio y aportaciones propias. Por ejemplo, cuando se dice que la sociología de la vejez no está presente en los debates sobre los problemas asociados al envejecimiento de nuestras sociedades, se puede estar reconociendo que realmente la sociología de la vejez no aporta nada a esos debates, o que sí lo hace, pero su aportación no se ve: "las iniciativas de estudio que se llevan a cabo para analizar y comprender las realidades sociales vinculadas al fenómeno de la vejez no se suelen traducir en transformaciones estructurales que podrían mejorar progresivamente las condiciones de vida de todas las personas mayores" (Experto 4).

El concepto de invisibilidad trata de aclarar cuál de esas dos posibilidades sucede realmente. Vistas las opiniones de los panelistas, parece que la más cierta es la segunda: la sociología de la vejez cuenta con discursos pero estos no se abren paso a la luz pública -incluso, a veces, ni siquiera dentro del mismo ámbito académico-.

Ya hemos adelantado que, según los datos de las tablas 6,7 y 8 , la amenaza más evidente identificada tiene que ver con el objeto de estudio de la sociolo-

Tabla 7

La invisibilidad como amenaza

\section{Amenazas futuras relacionadas con la invisibilidad de la} sociología de la vejez (W Kendall: $\mathbf{0 , 5}$ )

\begin{tabular}{|c|c|c|c|}
\hline & $\mathrm{n}$ & Media & $\begin{array}{c}\text { Desviación } \\
\text { típica }\end{array}$ \\
\hline $\begin{array}{l}\text { La resistencia de los viejos } \\
\text { a ser tomados como tales } \\
\text { hace que la sociología de la } \\
\text { vejez sea percibida como un } \\
\text { estudio algo negativo y que } \\
\text { hay que disimular. }\end{array}$ & 12 & 4,67 & ,888 \\
\hline $\begin{array}{l}\text { La Sociología de la Vejez } \\
\text { es una disciplina absorbida } \\
\text { por otras. }\end{array}$ & 12 & 3,42 & 1,311 \\
\hline $\begin{array}{l}\text { Ignorancia y desconocimiento } \\
\text { por parte de la sociedad en } \\
\text { general hacia la sociología } \\
\text { de la vejez, sus profesionales } \\
\text { y sus discursos, que han } \\
\text { penetrado escasamente. }\end{array}$ & 12 & 2,08 & 1,311 \\
\hline $\begin{array}{l}\text { Con los nuevos grados y la } \\
\text { adaptación al Espacio Europeo } \\
\text { de Educación Superior, este } \\
\text { campo no parece haber } \\
\text { logrado gran presencia. }\end{array}$ & 12 & 2,92 & ,793 \\
\hline $\begin{array}{l}\text { Otros campos, tanto dentro } \\
\text { de la Sociología (juventud, } \\
\text { desempleo, inmigración, } \\
\text { género, etc.) como en áreas } \\
\text { vecinas más asentadas } \\
\text { (Psicología de la Vejez, } \\
\text { Enfermería Geriátrica, } \\
\text { Pedagogía Gerontológica, } \\
\text { etc.), son considerados más } \\
\text { prioritarios. }\end{array}$ & 12 & 1,92 & ,793 \\
\hline
\end{tabular}

Fuente: Elaboración propia 
Tabla 8 .

El desinterés como amenaza

\begin{tabular}{lccc}
\hline \multicolumn{3}{c}{$\begin{array}{c}\text { Amenazas futuras relacionadas con el desinterés en } \\
\text { torno a la sociología de la vejez (W Kendall: 0,5) }\end{array}$} \\
\hline & $\mathrm{n}$ & Media & $\begin{array}{c}\text { Desviación } \\
\text { típica }\end{array}$ \\
\hline $\begin{array}{l}\text { Ni a los mismos viejos les } \\
\text { interesa la expansión de } \\
\text { este campo. }\end{array}$ & 12 & 3,83 &, 389 \\
\hline $\begin{array}{l}\text { La desidia, explícita } \\
\text { o implícita, de los } \\
\text { responsables universitarios } \\
\text { hacia este campo. }\end{array}$ & 12 & 2,17 & 1,030 \\
\hline $\begin{array}{l}\text { Declive del interés político } \\
\text { y mediático en torno al } \\
\text { envejecimiento, entre otras } \\
\text { razones, por estimar que } \\
\text { ocuparse de los viejos no es útil } \\
\text { ni productivo para la sociedad. }\end{array}$ & & & \\
\hline $\begin{array}{l}\text { Los jóvenes sociólogos no } \\
\text { están interesados en el } \\
\text { campo, quizá por la falta de } \\
\text { expectativas profesionales de } \\
\text { la Sociología en general. }\end{array}$ & & & \\
\hline
\end{tabular}

Fuente: Elaboración propia.

gía de la vejez: el enorme peso de la idea de vejez como declive -"cuerpo a extinguir"- parece colocar el estudio de la vejez en vía muerta y desvía a los investigadores hacia campos que se ocupan de grupos o fases del ciclo vital en promoción: "[La amenaza consiste en] Que disminuya la atención a este colectivo por estimar que ya no es útil ni productivo para la sociedad" (Experto 8).

¿Tiene esta amenaza algo que ver con la forma en la que la propia sociología de la vejez española ha sido capaz de concretar su objeto de estudio? Posiblemente sí: "La sociología de la vejez en España incorpora seguramente las debilidades teóricas de la sociología de la vejez en general, centradas acaso en los problemas de delimitación del objeto de estudio" (Experto 10).

El peso negativo de tal consideración deficitaria de la vejez también parece tener su impacto sobre las personas de más edad, en quienes supuestamente este campo se centra de modo prioritario. Los panelistas reconocen "resistencia" y desinterés por parte de las personas más envejecidas, que no desean prestarse a ser consideradas como objeto de estudio en tales condiciones de desconsideración. Esta "rebelión del objeto" es una cuestión a no dejar de lado. Y cabría preguntarse, con conciencia reflexiva, hasta qué punto no ha sido la misma deriva de la sociología de la vejez -la española en este caso- la que ha podido coadyuvar en la aparición de tales resistencia y desinterés. Sabemos que la caracterización de las personas mayores como grupo social distinto ha podido abrir la puerta a procesos de exclusión que, paradójicamente, más tarde se han convertido en asunto de interés y de denuncia sociológicos (Sánchez y
Díaz 2009): "[Constituye una amenaza] El despilfarro social de excluir a los mayores en diferentes ámbitos y oportunidades de la vida" (Experto 8).

Antes, ya nos hicimos eco brevemente de lo llamativo que resulta que los expertos esgriman el hecho de que "de la vejez todo el mundo se atreve a hablar" como importante amenaza para el campo de la sociología de la vejez. En principio, este mismo criterio se podría aplicar, por ejemplo, a otras sociologías como las de la comunicación, la familia, o el género, temas sobre los que podríamos presumir que, hasta cierto punto, todo el mundo es capaz de pronunciarse. ¿Por qué entonces este énfasis y el fuerte grado de consenso en torno a esta idea considerada como amenaza? La clave parece estar más bien en que ese "todo el mundo" se refiere, no a cualquier persona, sino a investigadores de otras disciplinas, como de hecho se reconoce en el apoyo de nuestros expertos al segundo enunciado de la Tabla 7: "La principal amenaza en mi opinión es el hecho de que sea una disciplina absorbida por otras" (Experto 11).

Esto último sí parece estar en línea con los análisis que repasamos más arriba en los que se aludía a la absorción -por acaparación de campos de estudio- que la gerontología, por ejemplo, ha ido realizando a su favor y en detrimento de la sociología de la vejez. Esta amenaza conduce a una cuestión que nos parece central: ¿cuál es la manera de hablar de la vejez propia y distinta de la sociología, esa manera que no estaría al alcance de "todo el mundo" ni de quienes se acercan a su estudio desde otros campos disciplinares? Y, si existe esa manera, ¿qué ha sucedido para que no llegue a ser objeto de suficiente atención ni logre mayor estatus tanto en la comunidad científica sociológica como con respecto a otras disciplinas y a la sociedad en general? Estas preguntas invitan a reconducir la discusión sobre las amenazas, no hacia afuera, sino hacia dentro del propio campo, como, de hecho, lo hizo uno de los expertos: "Las amenazas están dentro de nuestra propia ciencia y profesión, hay que convencer a nuestro entorno, a nuestros colegas, que esta subdisciplina es tan digna como aquellas más clásicas y que merece la pena poner atención en ella, así como apoyos de diferente índole" (Experto 2).

Tras las amenazas, el segundo tema en torno al cual se creó un cierto consenso -moderado, en este caso- fue el de las oportunidades de futuro para la sociología de la vejez; en términos cuantitativos el grado de acuerdo ( $W$ de Kendall) pasó de 0,3 en el segundo cuestionario a 0,5 en el tercero. En concreto, nuestros expertos se pusieron más de acuerdo a la hora de valorar positivamente las oportunidades relacionadas con el interés actual de la temática del campo. En la investigación, el término "interés" -interpretado como carencia del mismo- apareció fundamentalmente vinculado al de invisibilidad: la falta de interés podría interpretarse como una de las ra- 
zones de la invisibilidad, o viceversa, precisamente porque la sociología de la vejez es invisible no es capaz de suscitar interés. Sin embargo, como veremos a continuación, los expertos consultados consideran que, en general, y visto el devenir demográfico de la sociedad española, la vejez sigue siendo un asunto capaz de atraer el interés público -la carencia anterior es ahora sustituida por la existencia de interés-. De ahí, el carácter que ese interés externo tiene de oportunidad para la sociología de la vejez. La Tabla 9 muestra, colocados por orden de importancia, los tres enunciados que, de manera conjunta, fueron objeto de consenso moderado al respecto:

Tabla 9.

El interés como oportunidad

\begin{tabular}{|c|c|c|c|}
\hline \multicolumn{4}{|c|}{$\begin{array}{l}\text { Oportunidades futuras de la sociología de la vejez } \\
\text { relacionadas con el interés suscitado en torno a ella (W } \\
\text { Kendall: } 0,5)\end{array}$} \\
\hline & $\mathrm{N}$ & Media & $\begin{array}{c}\text { Desviación } \\
\text { típica }\end{array}$ \\
\hline $\begin{array}{l}\text { El interés investigador en torno } \\
\text { al envejecimiento es creciente } \\
\text { en cuanto al número de } \\
\text { universidades, de centros de } \\
\text { investigación y de sociólogos. }\end{array}$ & 12 & 2,67 & ,492 \\
\hline $\begin{array}{l}\text { Es precisamente este contexto } \\
\text { de crisis el que puede hacer } \\
\text { que resurja el interés y la } \\
\text { necesidad de conocer mejor } \\
\text { todos los aspectos que } \\
\text { afectan a una sociedad que } \\
\text { envejece imparablemente y en } \\
\text { la que se cuestiona el estado } \\
\text { del bienestar. }\end{array}$ & 12 & 2,08 & ,793 \\
\hline $\begin{array}{l}\text { El envejecimiento progresivo } \\
\text { de la población española y la } \\
\text { necesidad de indagar sobre } \\
\text { la evolución de la situación } \\
\text { sociodemográfica actual, } \\
\text { conducen a que los problemas } \\
\text { que son objeto de estudio } \\
\text { de la sociología de la vejez } \\
\text { constituyan un ámbito de } \\
\text { investigación social prioritario. }\end{array}$ & 12 & 1,25 & ,452 \\
\hline
\end{tabular}

Fuente: Elaboración propia.

No se puede soslayar el carácter a primera vista contradictorio de una consideración del interés como amenaza seguida de otra en la que se percibe como oportunidad. No obstante, la contradicción se aclara cuando se comprueba quiénes son los distintos agentes que están detrás de uno u otro interés. En el caso de la falta de interés, considerada como amenaza, los expertos habían aludido al desinterés de las personas mayores, de los responsables universitarios, de algunos agentes políticos y mediáticos, y de los jóvenes sociólogos. Ahora, el interés existente en torno a los temas de la vejez y el envejecimiento se le atribuye principalmente a los investigadores y, de modo difuso y general, a la misma sociedad: "El interés que para la sociedad debe de representar todo lo relacionado con un sector de la población, sumamente heterogéneo y con un incremento progresivo y constante de sus componentes" (Experto 8). No obstante, uno de los expertos fue más concreto al respecto: "En España se percibe un creciente interés por las instituciones, universidades, asociaciones, medios de comunicación y ciudadanía en general [...] por conocer más sobre estos temas que a todos incumben. Por ejemplo, se ofrecen varios postgrados [...] vinculados con el envejecimiento; hace años apenas se ofertaba alguno puntual. Varios programas comunicativos (radio, prensa, campañas publicitarias, etc.) actualmente destinan mayor espacio al perfil de personas mayores, a esta etapa vital y al estudio sobre los mismos" (Experto 13).

El interés existe. Lo que no parece evidente, a la luz de los testimonios de nuestro grupo de expertos, es que la sociología de la vejez española esté, hoy por hoy, aprovechando esta oportunidad. Y en la investigación no se aclaró de forma precisa y suficientemente consensuada qué obstáculos concretos están impidiendo tal proceso. Eso sí, se nombraron debilidades -cuya jerarquización solo logró un consenso débil (W Kendall: 0,3)- como las siguientes:

- Dificultad para delimitar el objeto de estudio propio de la sociología de la vejez.

- El campo está demasiado centrado en la fragilidad y la vulnerabilidad.

- Una equivocada consideración de que a este campo le corresponde estudiar a las personas de 65 o más años.

- La falta de claridad acerca de lo que se entiende por vejez dificulta el avance del campo y su reconocimiento.

- El campo está demasiado impregnado por la demografía.

- El campo, como especialidad académica, aún es inexistente en muchas universidades españolas, tanto a nivel de docencia como de investigación.

- La presencia del campo en muchos foros (por ejemplo, congresos, másteres o cursos especializados) aparece a menudo difuminada, difusa y/o compartiendo espacio con otros campos.

- Falta de penetración del campo en sociedades científicas nacionales e internacionales de fuerte incidencia social (por ejemplo, las dedicadas a la geriatría).

- El carácter transversal del campo trae consigo, en muchas ocasiones, una menor presencia del mismo.

- Falta de peso de este campo en la agenda pública. 
El último aspecto objeto de consenso moderado tuvo que ver con las fortalezas de la sociología de la vejez. En concreto, el grupo de expertos se mostró moderadamente de acuerdo en que algunos rasgos de la comunidad de agentes (investigadores, docentes y sociólogos/as profesionales) implicados más directamente en este campo constituyen activos importantes del mismo. En la Tabla 10, que mostramos a continuación, se detallan, por orden de importancia cuáles fueron esas características identificadas como fortalezas:

Tabla 10.

\section{La comunidad, una fortaleza}

Fortalezas relacionadas con la comunidad de agentes integrantes de este campo: investigadores, docentes y sociólogos/as profesionales (W Kendall: 0,5)

\begin{tabular}{lccc}
\hline & $N$ & Media & $\begin{array}{c}\text { Desviación } \\
\text { típica }\end{array}$ \\
\hline $\begin{array}{l}\text { Conocer a las personas } \\
\text { que formamos parte de } \\
\text { este campo. }\end{array}$ & 12 & 3,67 &, 651 \\
\hline $\begin{array}{l}\text { Incorporación progresiva } \\
\text { al campo de personas más } \\
\text { jóvenes. }\end{array}$ & 12 & 2,67 &, 492 \\
\hline $\begin{array}{l}\text { Presencia de la sociología } \\
\text { de la vejez en congresos, } \\
\text { seminarios y otras actividades } \\
\text { de difusión. }\end{array}$ & 12 & 2,00 & 1,128 \\
\hline $\begin{array}{l}\text { Contar en el campo con } \\
\text { miembros con una larga } \\
\text { trayectoria en el mismo. }\end{array}$ & 12 & 1,67 &, 985 \\
\hline
\end{tabular}

Fuente: Elaboración propia

Aunque pueda parecer contradictorio, el reducido tamaño del grupo de personas implicadas en la sociología de la vejez española, considerada como campo académico, se considera una fortaleza: "[Una fortaleza son los] Intercambios entre los investigadores y docentes, todos nos conocemos y renovamos frecuentemente nuestra relación en la participación a congresos" (Experto 5). Y esta fortaleza ha tenido un proceso de gestación bastante reciente: "A lo largo de un par de décadas, de mediados de los ochenta a mediados de los años dos mil, pero, con el centro, insisto, en los noventa, todo los que investigamos nos conocemos e intercambiamos nuestros conocimientos y nos invitamos a participar en las respectivas publicaciones" (Experto 5). No obstante, a la luz de lo afirmado por algunos de nuestros panelistas, este conocimiento debería dar paso a algo mejor aún: "Un grupo de personas que quisieran hacer cosas en común y [a las que] les ilusione trabajar juntos" (Experto 12), o bien "Que los sociólogos/as interesados en el tema mantengan vivo y encendido siempre el interés por la ampliación y perfeccionamiento de ese conocimiento puede llevar a, no solo mantener la llama viva entre los seniors sino, también, a contagiar el entusiasmo a los jóvenes" (Experto 7).
Por último, hay que aludir a otra aparente contradicción: si más arriba se decía que una amenaza consiste en que los jóvenes sociólogos no están interesados en el campo, quizá por la falta de expectativas profesionales de la sociología en general, ahora se habla de la incorporación de algunas personas más jóvenes, lo que permite conservar una cierta esperanza de cara al futuro, visión claramente en línea con el diagnóstico realizado para el caso norteamericano (Bengtson 2011): "La consolidación de redes y la mayor trayectoria de investigadores en este campo abren oportunidades de difusión de la investigación y de formación de jóvenes con proyección de futuro" (Experto 3).

Finalizamos la revisión de los resultados de la investigación con una alusión a las estrategias. $Y$ lo hacemos con dos aclaraciones previas. Por un lado, ninguno de los seis aspectos valorados por nuestros expertos a modo de grupos de estrategias posibles alcanzó el umbral cuantitativo suficiente de consenso. Sin embargo, por otro lado, también es cierto que uno de esos seis aspectos -lo que investigadores, docentes y sociólogos profesionales implicados en la sociología de la vejez deberían hacer- quedó muy cerca de lograrlo: su valor final de $W$ de Kendall fue de 0,45 . No obstante, hemos decidido hacernos eco de aquellas iniciativas que, según los panelistas, y tras meses de implicación en el estudio, habría que llevar a cabo para impulsar la sociología de la vejez en España; creemos que esta información puede resultar especialmente interesante para quienes se acerquen a la lectura de este artículo con la intención de conocer iniciativas que se podrían poner en práctica.

En concreto, los expertos participantes en el estudio plantearon -en orden de mayor a menor importancia- las siguientes cuatro acciones relacionadas con la comunidad de agentes (investigadores, docentes y sociólogos profesionales) que componente este campo académico:

- Mantener siempre vivo y encendido el interés por la ampliación y el perfeccionamiento del conocimiento, lo que puede llevar, no solo a mantener la llama viva entre los senior, sino también a contagiar el entusiasmo a los jóvenes.

- Se necesitaría que alguien con muchas ganas de dinamizar la especialidad se encargara de hacerlo. En ese sentido, sería importante fomentar los equipos de investigación en las distintas universidades y los equipos de investigación conjuntos e incrementar el número de proyectos de investigación.

- Empeñarnos en realizar tareas de calidad, buscando colaboraciones efectivas con otras personas y grupos, y sometiendo nuestros trabajos de investigación a revisiones externas de reconocido prestigio internacional -envian- 
do nuestros trabajos, por ejemplo, a las mejores revistas científicas-.

- Aumentar la comunicación entre grupos de investigación y el contacto con países y centros de investigación que ofrecen oportunidades para formarse e investigar.

\section{Conclusiones}

La técnica Delphi ha demostrado ser un procedimiento útil para facilitar la discusión y la creación de consenso en torno al estatus presente y a las perspectivas futuras de la sociología de la vejez española. Nuestro grupo de expertos se ha puesto de acuerdo, en primer lugar, en que, en España, la sociología de la vejez adolece, sobre todo, de varias amenazas; es decir, de elementos externos a este campo disciplinar y académico que lo han colocado en situación de peligro y que atentan contra su existencia y desarrollo futuros. En concreto, esas amenazas son un cierto agotamiento de la vejez como objeto de estudio -en paralelo con la negación de un número creciente de personas mayores a ser encuadradas dentro de la etapa de la vejez y con un abierto intrusismo por el hecho de que, de la vejez, todo el mundo se atreve a hablar-, la invisibilidad pública de las aportaciones de la sociología de la vejez y, por último, el desinterés externo hacia este campo en favor de una mayor atención a otros grupos de edad y otras fases del ciclo vital. Desde este punto de vista, la conclusión general del estudio tiene un tono pesimista y confirma que estamos ante un campo aún sin establecer plenamente dentro de la Sociología académica.

No obstante, en segundo lugar, los doce panelistas han coincidido en que la sociología de la vejez en España aún tiene por delante una buena oportunidad: el interés genérico de la sociedad y el interés específico de algunos investigadores en torno a los procesos de envejecimiento, que siguen ocupando un cierto espacio en las agendas públicas y políticas. En tercer lugar, la impresión del grupo de expertos participantes ha sido que existe una comunidad compuesta por investigadores, docentes y sociólogos profesionales que se conocen entre

\section{Referencias Bibliográficas}

Acevedo-Polakovich, I. D., B. Bell, P. Gamache and A. S. Christian. 2013. "Service Accessibility for Lesbian, Gay, Bisexual, Transgender, and Questioning Youth". Youth \& Society 45(1):75-97. https://dx.doi. org/10.1177/0044118X11409067

Alfageme, A., B. García y C. Viñado. 2014. "El retiro temporal del trabajo remunerado como alternativa a la jubilación. Algunas opiniones expertas". Papers 99: 161-186. https://dx.doi.org/10.5565/rev/papers.583

Alkema, G. E. y D. E. Alley. 2006. “Gerontology's Future: An Interpretive Model for Disciplinary Advancement". The Gerontologist 46: 574-582. https://dx.doi.org/10.1093/ geront/46.5.574 sí, que cuentan con una larga trayectoria en este campo y que constituyen una fortaleza en la que apoyarse de cara al futuro.

En conjunto, el panorama es ambivalente: la constatación de la existencia de evidentes amenazas convive con la sensación de que se dispone de condiciones externas -el interés que suscita el envejecimiento-, e internas -la comunidad de agentes instalados en el campo- que permiten vislumbrar posibilidades de desarrollo de la sociología de la vejez en el futuro. Para que ese desarrollo tenga lugar, nuestros expertos recomiendan ejecutar estrategias como la producción de conocimiento de mayor calidad, la dinamización y el aumento de la colaboración entre los miembros del campo, o una mejor comunicación nacional e internacional entre los grupos de investigación dedicados a temáticas propias de la sociología de la vejez.

Lo que ha quedado sin responder es quiénes y cómo podrían echar a andar tales estrategias. Quizá la lectura del presente trabajo pueda servir para suscitar un cruce de impresiones al respecto y, por qué no, emprender algún tipo de acción, si bien esto último va más allá de los límites que nuestro estudio Delphi se había marcado.

Reconocemos que haber logrado la implicación de un mayor número de expertos habría enriquecido más la diversidad de las reflexiones y los argumentos $\mathrm{y}$, por ende, las posibilidades de consenso, pero, a la vez, el método adoptado exigía respetar escrupulosamente los criterios de localización e inclusión fijados. Futuros trabajos podrían intentar, no solo contar con más expertos sino, incluso barajar la conveniencia de un panel abiertamente multidisciplinar. Lo que parece indudable es que, tal y como sostenían Angel y Settersten (2011), en los próximos años los sociólogos españoles dedicados a la vejez tendrán que continuar enfrentándose a la tarea de concretar cuál es la verdadera singularidad de su campo cuando el carácter multidisciplinar de las investigaciones sociales sobre el envejecimiento vaya en aumento. Prestar atención a las amenazas, oportunidades y fortalezas señaladas en este trabajo puede ser una forma de empezar a acometer tal tarea.

Angel, J. L. y R. A. Settersten. 2011. "Sociology of Aging in the Decade Ahead". Pp. 661-672 en Handbook of Sociology of Aging, editado por R. Settersten y J. L. Angel. New York, NY: Springer Science+Bussiness Media.

Atkins, R. B., H. Tolson and B. R. Cole. 2005. "Stability of response characteristics of a Delphi panel: application of bootstrap data expansion". BMC Medical Research Methodology 5: 37. https://dx.doi.org/10.1186/14712288-5-37

Barrett, A. E., R. Redmon y C. von Rohr. 2012. "Avoiding Aging? Social Psychology's Treatment of Age". American Sociologist 43: 328-347. https://dx.doi.org/10.1007/ s12108-012-9157-2 
Bazo, M. T. 1996. "Presentación”. Revista Española de Investigaciones Sociológicas 73: 9-13.

Bengtson. V. L. 2011. "Gerontology with a 'J': Personal Reflections on Theory-Building in the Sociology of Aging". Pp. 619-626 en Handbook of Sociology of Aging, editado por R. Settersten y J. L. Angel. New York, NY: Springer Science+Bussiness Media.

Bengtson, V. L., E. O. Burgess y T. M. Parrott. 1997. "Theory, Explanation, and a Third Generation of Theoretical Development in Social Gerontology". The Journals of Gerontology: SOCIAL SCIENCES 52B: S72-S88. https://dx.doi.org/10.1093/geronb/52B.2.S72

Bergman, E. M. L. 2011. "Social Gerontology-Integrative and Territorial Aspects: A Citation Analysis of Subject Scatter and Database Coverage". Behavioral \& Social Sciences Librarian 30: 154-175. https://dx.doi.org/10.108 $0 / 01639269.2011 .592803$

Bramwell, R. D. 1985. "Gerontology as a Discipline". Educational Gerontology 11: 201-211. https://dx.doi. org/10.1080/0380127850110403

Braun, V. y V. Clark. 2006. "Using Thematic Analysis in Psychology". Qualitative Research in Psychology 3: 77101. https://dx.doi.org/10.1191/1478088706qp063oa

Brink, P. J. 1987. "On Reliability and Validity in Qualitative Research". Western Journal of Nursing Research 9: 157-159.

Burawoy, M. 2005. "For Public Sociology". American Sociological Review 70: 4-28. http://dx.doi. org/10.1177/000312240507000102

Bush, G. y J. L. Jones. 2010. "Exploration to Identify Professional Dispositions of School Librarians: A Delphi Study". School Library Research 13: 1-22.

Donohoe, H. M. y R. D. Needham. 2009. "Moving Best Practice Forward: Delphi Characteristics, Advantages, Potential Problems, and Solutions". International Journal of Tourism Research 11: 415-437. https://dx.doi.org/10.1002/jtr.709

Ferri, C. P., M. Prince, C. Brayne, H. Brodaty, L. Fratiglioni, M. Ganguli, M. Scazufca. 2006. "Global prevalence of dementia: a Delphi consensus study". Lancet 366: 211217. https://dx.doi.org/10.1016/S0140-6736(05)67889-0

Federación Española de Sociología (FES). s.f. GT17 Sociología de la Edad y Ciclo Vital. Consulta 23 de febrero de 2015 (http://www.fes-sociologia.com/gt-17-sociologia-de-laedad-y-ciclo-vital/pages/167/).

Graneheim, U. H. y B. Lundman. 2004. "Qualitative content analysis in nursing research: Concepts, procedures and measures to achieve trustworthiness". Nurse Education Today 24: 105-112. https://dx.doi.org/10.1016/j. nedt.2003.10.001

Harris, J., J. Pryor y S. Adams. 1997. The Challenge of Intercoder Agreement in Qualitative Inquiry. Manuscrito no publicado, University of Texas. Consulta 23 de febrero de 2015 (https://www.researchgate.net/profile/ Judith_Harris4/publication/228490436_The_challenge_of_intercoder_agreement_in_qualitative_inquiry/ links/0c960527a47d4845d1000000.pdf).

Hohn, R. 2010. Transition to end-of-life in the Aged Care Setting: Identifying the Changes. Tesis de Máster, School of Nursing and Midwifery, Faculty of Health Sciences, Australian Catholic University. Consulta 23 de febrero de 2015 (http://trove.nla.gov.au/version/190025779).

Hruschka, D. J., D. Schwartz, D. Cobb, E. Picone-Decaro, R. A. Jenkins y J. W. Carey. 2004. "Reliability in Coding Open-Ended Data: Lessons Learned from HIV Behavioral Research". Field Methods 16: 307-331. https:// dx.doi.org/10.1177/1525822X04266540

Hsu, C. y B. A. Sandford. 2007. "The Delphi Technique: Making Sense of Consensus". Practical Assessment Re- search \& Evaluation 12: 1-8. Consulta 23 de febrero de 2015 (http://pareonline.net/pdf/v12n10.pdf).

Huenchuan, S. 2009. Escenarios futuros en políticas de vejez: Estudio Delphi comparado entre países seleccionados. Santiago de Chile: Naciones Unidas.

Jeste, D., M. Ardelt, D. Blazer, H. C. Kraemer, G. Vaillant y T. W. Meeks. 2010. "Expert Consensus on Characteristics of Wisdom: A Delphi Method Study". The Gerontologist 50: 668-680. https://dx.doi.org/10.1093/geront/gnq022

Landis, J. R. y G. G. Koch. 1977. "The measurement of observer agreement for categorical data". Biometrics 33: 159-174. Consulta 25 de febrero de 2015 (http://www. jstor.org/stable/2529310).

Katz, S. 1996. Disciplining Old Age: The Formation of Gerontological Knowledge. Charlottesville: University Press of Virginia.

Lowenstein, A. 2004. "Gerontology Coming of Age: The Transformation of Social Gerontology into a DistinctAcademic Discipline". Educational Gerontology 30: 129-141. http://www. tandfonline.com/doi/abs/10.1080/03601270490266293

Marshall, V. W. and V. L. Bengtson. 2011. "Theoretical Perspectives on the Sociology of Aging". Pp. 17-33 en Handbook of Sociology of Aging, editado por R. Settersten y J. L. Angel. New York, NY: Springer Science+Bussiness Media.

Nieto, J. A. 1981. "En torno a 'Perspectiva sociológica de la vejez"'. Revista Española de Investigaciones Sociológicas 14: 113-118. Consulta 16 de febrero de 2015 (http://www.jstor.org/stable/40182869).

Okoli, C. and S. D. Pawlowski. 2004. "The Delphi method as a research tool: an example, design considerations and applications". Information \& Management 42: 15-29. https://dx.doi.org/10.1016/j.im.2003.11.002

Phillipson, C. y J. Baars. 2007. "Social theory and social ageing". Pp. 68-84 en Ageing in Society, editado por J. Bond, S. Peace, F. Dittmann-Kohli y G. Westerhof. London: Sage.

Pinazo, S. y M. Sánchez. 2005. "Claves para comprender la posición actual de la Gerontología". Pp. 3-34 en Gerontología. Actualización, innovación y propuestas, dirigido por S. Pinazo y M. Sánchez. Madrid: PearsonPrentice Hall.

Powell, J. L. and J. Hendricks, J. 2009. "The Sociological Construction of Ageing: Lessons for Theorizing". International Journal of Sociology \& Social Policy 29: 84-94. https://dx.doi.org/10.1108/01443330910934745

Putney, N. M., D. E. Alley and V. L. Bengtson. 2005. "Social gerontology as public sociology in action". The American Sociologist fall/winter: 88-104. Consulta 26 de febrero de 2015 (http://link.springer.com/article/10.1007/ s12108-005-1018-9\#page-1).

Richardson, S. K. 2011. Defining appropriateness of Emergency Department attendance: a NZ perspective. Tesis doctoral, University of Otago. Consulta 23 de febrero de 2015 (http:// otago.ourarchive.ac.nz/bitstream/handle/10523/2469/RichardsonSandraK2011PhD.pdf?sequence=1).

Riley, M. W., Johnson, M. y A. Foner. 1972. Aging and Society. Vol III: A Sociology of Age Stratification. New York: Russell Sage Foundation.

Rodríguez, J. E. 1979. "Perspectiva sociológica de la vejez". Revista Española de Investigaciones Sociológicas 7: 77-97. Consulta 23 de febrero de 2015 (http://www. jstor.org/stable/40182762)

Sahin, A. E. 2010. "Professional status of elementary teaching in Turkey: a Delphi study". Teachers and Teaching: Theory and Practice 16: 437-459. http://www.tandfonline.com/doi/full/10.1080/13540601003754822 
Sánchez, M. y P. Díaz. 2009. "Análisis sociológico de la vejez en las sociedades occidentales". Pp. 13-44 en La ancianidad en nuestro mundo: más allá de los tópicos, coordinado por J.C. Jiménez y F. Torralba. Barcelona: Prohom Edicions.

Saldaña, J. 2009. The coding manual for qualitative researchers. London: Sage.

Schmidt, R. C. 1997. "Managing Delphi Surveys Using Nonparametric Statistical Techniques". Decision Sciences 28: 763-774. https://dx.doi.org/10.1111/j.1540-5915.1997. tb01330.x

Settersten, R. A. y J. L .Angel. (Eds.). 2011a. Handbook of Sociology of Aging. New York, NY: Springer Science+Bussiness Media.
Settersten, R. A. y J. L .Angel. 2011b. "Trends in the Sociology of Aging: Thirty Year Observations". Pp. 3-13 en Handbook of Sociology of Aging, editado por R. Settersten y J. L. Angel. New York, NY: Springer Science+Bussiness Media.

Walker, J. 2011. "Being professional in English language teaching services: a Delphic panel study". Quality Assurance in Education 19: 307-334. https://dx.doi. org/10.1108/09684881111170050

Wittman, H., M. Beckie and C. Hergesheimer. 2012. "Linking Local Food Systems and the Social Economy? Future Roles for Farmers' Markets in Alberta and British Columbia". Rural Sociology 77: 36-61. https://dx.doi. org/10.1111/j.1549-0831.2011.00068.x

MARIANO SÁNCHEZ MARTíNEZ es Profesor Titular de Sociología de la Universidad de Granada, investigador afiliado del Center for Healthy Ageing (Penn State University) y coordinador del Certificado Europeo en Aprendizaje Intergeneracional. Doctor en Sociología por la Universidad de Granada, sus tareas de investigación están centradas en el envejecimiento activo y en el análisis de las relaciones y programas intergeneracionales.

JUAN LÓPEZ DOBLAS es Doctor en Sociología por la Universidad de Granada y Profesor Titular de Universidad en el Departamento de Sociología (Facultad de Ciencias Políticas y Sociología) de dicha Universidad. Premio IMSERSO Infanta Cristina 2004 en 'Estudios e Investigaciones Sociales'. Principales líneas de investigación: Sociología del Envejecimiento y Sociología de la Familia. 